\title{
HEALTH-RELATED QUALITY OF LIFE OF HEALTH PROFESSIONALS IN A TERTIARY HEALTH INSTITUTION OF SOUTHWESTERN NIGERIA: A CROSS-SECTIONAL SURVEY
}

\author{
${ }^{* 1}$ Odetunde M. O., ${ }^{1}$ Ajibola B. O., ${ }^{2}$ Ilesanmi O.T., ${ }^{1}$ Okonji A. M., ${ }^{1}$ Ojoawo A. O., ${ }^{1}$ Awotidebe \\ T. O., ${ }^{3}$ Odetunde N. A., ${ }^{2}$ Omisore O. B. \& ${ }^{1}$ Mbada C. E.
}

\author{
${ }^{*}$ Department of Medical Rehabilitation, Faculty of Basic Medical Sciences, College of Health Sciences, Obafemi Awolowo University, \\ Ile-Ife, Osun state, Nigeria \\ ${ }^{2}$ Department of Physiotherapy, Obafemi Awolowo University Teaching Hospital, Ile-Ife, Osun state, Nigeria \\ ${ }^{3}$ General Out-patient Department, Wesley Guild Hospital, Ilesha, Osun State, Nigeria \\ *Corresponding Author Email: marodet2010@gmail.com Phone: +2347060809587
}

\section{ABSTRACT}

Introduction: Health professionals (HP) are frequently exposed to a high number of hazards at the workplace. Consequently, low ratings for their well-being and satisfaction with health care may adversely affect the quality of health services they provide. This study aimed to assess health-related quality of life (HRQOL) and determine the effects of socio-demographic variables on HRQOL across HP in a tertiary health institution in Nigeria, a low-middle-income economy. Method: This was a cross-sectional survey of 385 HP purposively recruited from different units of Obafemi Awolowo University Teaching Hospital (OAUTH), Ile-Ife, Nigeria. All respondents completed the Medical Outcomes Study short-form (SF-36) questionnaire. Also, the socio-demographic information on the respondents was obtained. Descriptive statistics of mean, standard deviation, frequency and percentages; and inferential statistics of independent t-test and one way ANOVA was used to analyze data. Alpha level was set at $p<0.05$. Results: Respondents comprised 273 males and 112 females. They scored well above

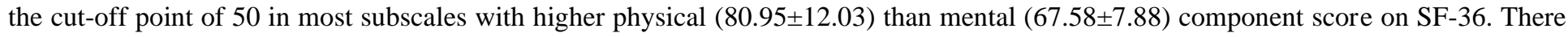
were significant differences between gender in favour of male respondents with the highest mean score across each of the age groups, educational and professional qualifications $(\mathrm{p}<0.01)$ on most subscales. Conclusion: There are considerable inequalities in HRQOL among HP in this study. These inequalities are gender-biased and favour professionals with a post-graduate degree. Findings from this study are comparable to other climes and advocate support for female HP, those on low cadre and higher degree training.

Keywords: Health professionals, health-related QOL, socio-demographic characteristics

LICENSE: This work by Open Journals Nigeria is licensed and published under the Creative Commons Attribution License 4.0 International License, which permits unrestricted use, distribution, and reproduction in any medium, provided this article is duly cited.

COPYRIGHT: The Author(s) completely retain the copyright of this published article.

OPEN ACCESS: The Author(s) approves that this article remains permanently online in the open access (OA) model.

QA: This Article is published in line with "COPE (Committee on Publication Ethics) and PIE (Publication Integrity \& Ethics)". 


\section{INTRODUCTION}

Health in the context of its relationship to the quality of an individuals' life with consideration for health and disease is termed health-related quality of life (Tountas et al, 2007). Health-related quality of life (HRQOL) is a subjective, multidimensional concept that comprises domains related to physical, mental, emotional, and social functioning and includes both positive and negative facets of life (Centre for Disease Control, 2021). Health professionals are a vulnerable group of workers who are frequently exposed to a high number of hazards at the workplace, thus their HRQOL is an important factor that determines the quality of health services they provide (Guler and Kuzu, 2009; Tinubu et al, 2010). Health-related quality of life has been extensively studied in patients with various diseases with emphasis on the impact of the disease on patients' HRQOL (Acaray and Pinar, 2004; Lee et al, 2006; Landman et al, 2010; Oni et al, 2016; Phan et al, 2019). On the contrary, HRQOL researches are limited among the healthy population, while this is important for assessing both positive and negative facets of life and how it affects individuals' health and service delivery, researches in this area started to evolve. Thus, there has been growing awareness of the need to assess the HRQOL of the general population and workers in the health sector (Palhares et al, 2014). Consequently, several studies on HRQOL in the general population and among health professionals in different settings have been conducted (Müller et al, 2007; Guler and Kuzu, 2009; Mujchin, 2015; Kumar et al, 2018; Barbosa et al, 2018) mainly in the developed system which suggested adverse outcome. This concept has however not been

well documented for HP in the health system of low-and-middle-income countries (LMIC) despite sub-Saharan Africa being known to have the lowest ratings for well-being and the lowest satisfaction with health care (Deaton and Tortora, 2015). Health professionals are constantly exposed to physical, chemical, biological, psychosocial and ergonomic hazards at the workplace, which increases the possibility of low quality of life. Little wonder they were selected as a priority for improvement of safety and health at work in the World Health Organization (WHO) Work Plan 20092012 and their continued relevance in WHO Work Plan of 2014-2019. Quality of life is assessed using standardized questionnaires developed by different entities with consideration for the multidimensional and personal perceptions nature of the QOL. The Medical Outcomes Study (MOS) Short Form 36-item Health Survey (SF-36), is one of the most widely used generic measures of HRQOL with good psychometric properties and substantial data on its applicability in clinical and research settings (ten Klooster et al, 2013). Assessing the pattern of HRQOL of health professionals in the LMIC using the Nigerian context, compared with other climes is the motivation for this study.

\section{AIMS AND OBJECTIVES}

This study aimed to assess HRQOL and determine the influence of socio-demographic and clinical variables (gender, age group, specialty, educational qualification and years in profession) across the HP groups in OAUTH. The study objectives were to assess the HRQOL of HP in OAUTH, compare HRQOL of the different HP groups and compare selected socio-demographic variables across the HP groups. 


\section{PURPOSE OF RESEARCH}

HRQOL among health professionals in different settings has been reported to be generally low even among those in a good work environment. While the case may not be better among HPs in sub-Saharan Africa who are known to have the lowest ratings for well-being and satisfaction with health care, it, therefore, becomes pertinent to have an idea of HRQOL of this population in this environment as this will help determine not only their health status but also the quality of care they deliver to the patients and how this compares with other climes.

\section{RESEARCH JUSTIFICATION}

Health professionals as adults have a vital influence on the health behaviours of their patients and clients with its consequences for diseases in the medium and long term in future generations (Noronha et al, 2016). Evidence exists that the work situation of health professionals adversely affects their quality of life and safety of care (Mc Vicar, 2003; Nylenna et al, 2005; Gurses et al, 2009). Good HRQOL of health professionals is thus important for the management of the hospitals and increasing the quality of health services they provide for their patients and clients (Oyama et al, 2015). Further studies of the HRQOL of health professionals would therefore be an important step that could lead to the development of policies to improve working conditions in hospitals (Oyama et al, 2015).

\section{HYPOTHESIS}

We hypothesized that scores of health professionals on SF-36 subscales would not differ significantly across the sociodemographic and clinical variables.

\section{METHODOLOGY}

\section{Research Design}

This was a cross-sectional survey.

\section{Sampling and sampling technique}

Respondents were purposively recruited from three hospital units of OAUTHC viz: Ife Hospital unit, Wesley Guild Hospital unit, Ilesha; and Urban Comprehensive Health Centre, Eleyele Ile-Ife which were purposively selected being the units with a greater number of HPs. All eligible HPs (Medical doctors, Medical Laboratory Scientists, Nurses, Pharmacists, Physiotherapists and Radiographers) who were approached and gave consent to respond to the questionnaire were recruited for the study. Inclusion criteria were being a current HP in any of the selected hospital units, while current staff of the units who are not HP were excluded from this survey.

\section{Respondents}

The sample size was calculated using the following survey sample size formula (Bartlett et al. (2001)

$$
n=\frac{p(100-p) z^{2}}{E^{2}}
$$


(Where $\mathrm{n}$ is the required sample size, $\mathrm{P}$ is the percentage occurrence of a state or condition (50\% as an estimate of $\mathrm{P}$ ), $\mathrm{E}$ is the percentage maximum error required (a $5 \%$ margin of error is acceptable), $\mathrm{Z}$ is the value corresponding to a level of confidence required, at 95\%; $\mathrm{Z}=1.96$ ). Giving a sample size of 384.16

The sample comprised 385 health professionals (168 Medical doctors, 32 Medical Laboratory Scientists, 73 Nurses, 53 Pharmacists, 18 Physiotherapists and 41 Radiographers). Respondents were staff of different hospital units of the Obafemi Awolowo University Teaching Hospital, (OAUTH) Ile Ife, Osun State, southwestern Nigeria.

\section{Instrument}

The SF-36 was used to assess HRQOL of respondents in this study. The tool assesses eight health dimensions referred to as subscales, namely Physical Functioning (PF: 10 items), Role Limitations due to Physical Problems (RP: 4 items), Bodily Pain (BP: 2 items), General Health (GH: 5 items), Vitality (VT: 4 items), Social Functioning (SF: 2 items), Role Limitation due to Emotional Problems (RE: 3 items) and Mental Health (MH: 5 items). It also includes a single item that indicates perceived change in health to make a total of 36 items. This single item is not included in the scoring process. Reliability values are Cronbach's Alpha ranges from 0.78 to 0.93 on the subscales. The subscales' scores are summarized into Physical Component Summary (PCS) and Mental Component Summary (MCS). The PCS includes four subscales: PF, RP, BP and GH; the MCS also includes four subscales: VT, SF, RE and MH. Individual SF-36 items are recoded, summed and transformed. The health concepts described by the SF-36 range in score from $0-100$, with higher scores indicating higher levels of function or better health and a mean score of 50 has been set as a normative value for all scales. Scoring is a two-step process. First, pre-coded numeric values are recoded using the standard SF-36 scoring algorithms. In addition, each item is scored on a 0 to 100 range so that the lowest and highest possible scores are 0 and 100, respectively. Scores represent the percentage of the total possible score achieved. In step 2, items in the same scale are averaged together to create the 8 scale scores. Scores on the eight subscales can be used to compute a summary index of PCS and MCS respectively (Gandek et al, 2004; Müller et al, 2007).

\section{ETHICAL PROCEDURE}

The research was approved by the Health Research Ethics Committee, Institute of Public Health, Obafemi Awolowo University, Ile-Ife, with protocol number IPHOAU/12/1353. Written informed consent was sought and obtained from each of the respondents after the researcher explained the concept and procedure of the research. Anonymity was strictly observed. Respondents were assured of non-maleficent of the study and freedom to refuse to respond to the questionnaire or withdraw from the study at any stage without any penalty.

\section{PROCEDURE}

Following the ethical procedure, demographic and clinical data of respondents were obtained using a proforma. The SF-36 questionnaire was self-completed by each respondent in their different departments at the in-patient, out-patient clinics, offices and lounges in the presence of the researcher who collected the questionnaire immediately after completion. All questionnaires were duly completed and returned, giving a 100\% response rate. 


\section{DATA ANALYSIS}

Descriptive statistics of mean, standard deviation, frequency and percentages were used to summarize the sociodemographic and clinical variables of respondents. Inferential statistics of independent t-test was used for comparison of quantitative variables between two groups. For comparison of quantitative variables among more than two groups, one-way Analysis of Variance (ANOVA) was used. Data were analyzed using the Statistical Package for the Social Sciences (SPSS version 21). Alpha level was set at $\mathrm{p}<0.05$.

\section{RESULTS}

A total of 385 health professionals responded to the questionnaire giving a $100 \%$ response rate. The frequency distribution of HP is presented in figure 1. Majority of the respondents were medical doctors (43.6\%), males (65.5\%), in the 20-39-year age group (80\%) and hold a bachelor's degree (65.7). The distribution of respondents by profession is presented in figure 2. Slightly more than half of the respondents were married (52.8\%), a few were smokers (7.7\%) and fewer take alcohol (4.6\%). Other details are presented in Table I.

The Respondents scored well above the cut-off point of 50 in all subscales except VT and $\mathrm{MH}$; with the highest score on RP $(91.41 \pm 21.95)$ and lowest on VT (49.72 \pm 15.22$)$ subscales. The score on PCS $(80.95 \pm 12.03)$ was higher than MCS (67.58 \pm 7.88$)$ (Table II). Significant gender differences $(\mathrm{p}<0.05)$ occurred on five subscales, male respondents scored higher on three (GH, SF, RE) as well as MCS (Table II). There were significant differences across the age groups on PF, BP, GH, VT, SF, MH subscales and PCS $(\mathrm{P}<0.05$; partial eta squared ranged from 0.01 to 0.27). The highest mean scores in GH $(80.00 \pm 18.12)$ and SF $(89.61 \pm 16.84)$ domains of SF-36 were found among health professionals aged $20-29$ years $(p=0.001)$ (Table III). 


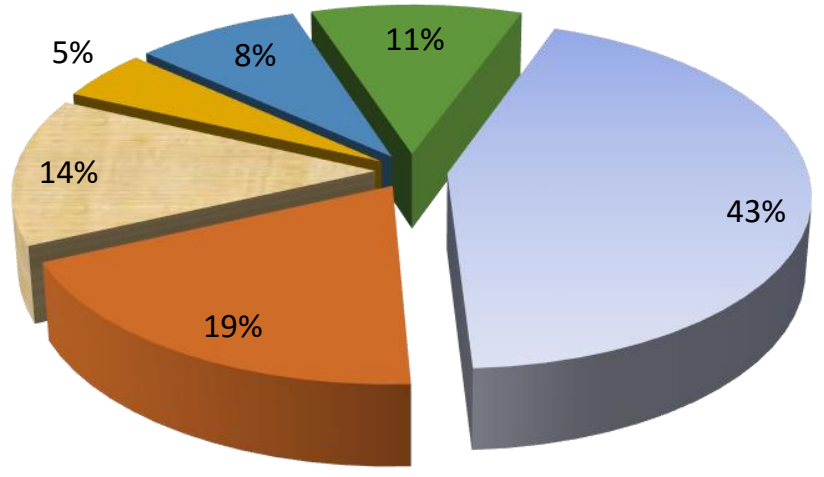

Medical doctors

Nurses

Pharmacists

Physiotherapist

Medical laboratory scientist

Radiographers

Figure 1: Frequency Distribution of Health Professionals

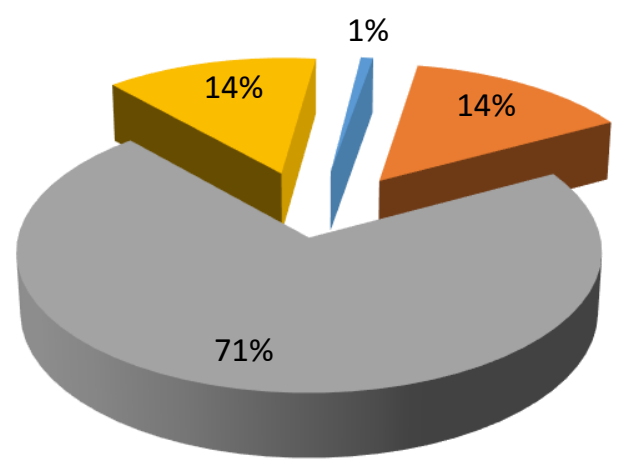

Registered Nurse/Midwife

Bachelor's Degree

Master's Degree

Ph. D

Figure 2: Distribution of Health Professionals by Educational Qualification 
Table I: Socio-demographic Characteristics of Respondents

\begin{tabular}{|c|c|c|}
\hline Variables & Frequency & Percentages \\
\hline \multicolumn{3}{|l|}{ Gender } \\
\hline Male & 273 & 70.9 \\
\hline Female & 112 & 29.1 \\
\hline \multicolumn{3}{|l|}{ Age } \\
\hline $20-29$ & 159 & 41.3 \\
\hline $30-39$ & 147 & 38.2 \\
\hline $40-49$ & 74 & 19.2 \\
\hline $50-59$ & 3 & 0.8 \\
\hline $60-69$ & 2 & 0.5 \\
\hline \multicolumn{3}{|l|}{ Marital status } \\
\hline Single & 164 & 42.6 \\
\hline Married & 221 & 57.4 \\
\hline \multicolumn{3}{|l|}{ Years in the profession } \\
\hline $0-10$ & 276 & 71.7 \\
\hline $11-20$ & 98 & 25.4 \\
\hline $21-30$ & 6 & 1.6 \\
\hline $31-40$ & 5 & 1.3 \\
\hline \multicolumn{3}{|l|}{ Grade level } \\
\hline $6-9$ & 213 & 55.3 \\
\hline $10-12$ & 125 & 32.5 \\
\hline $13-15$ & 47 & 12.2 \\
\hline \multicolumn{3}{|l|}{ Social habit } \\
\hline Takes alcohol & 19 & 4.9 \\
\hline Smokes & 32 & 8.3 \\
\hline Neither alcohol nor smokes & 334 & 86.8 \\
\hline \multicolumn{3}{|l|}{ Ethnicity } \\
\hline Igbo & 84 & 21.8 \\
\hline Yoruba & 289 & 75.1 \\
\hline Others & 12 & 3.1 \\
\hline \multicolumn{3}{|l|}{ Religion } \\
\hline Christianity & 331 & 86.0 \\
\hline Islam & 52 & 13.5 \\
\hline Others & 2 & 0.5 \\
\hline
\end{tabular}


Table II: Independent t-test comparison of respondents scores on SF-36 subscales by gender at 95\% CI

\begin{tabular}{llllll}
\hline Subscales & $\begin{array}{l}\text { Overall } \\
\overline{\mathbf{x}} \pm \text { SD } \\
(\mathbf{n = 3 8 5})\end{array}$ & $\begin{array}{l}\text { Male } \\
\overline{\mathbf{x}} \pm \mathbf{S D}(\mathbf{n}=\mathbf{2 7 3})\end{array}$ & $\begin{array}{l}\text { Female } \\
\overline{\mathbf{x}} \pm \text { SD } \\
(\mathbf{n = 1 1 2})\end{array}$ & t-calculated & P-value \\
& & & & & \\
\hline PF & $83.82 \pm 21.38$ & $83.19 \pm 22.5$ & $85.36 \pm 18.4$ & 0.904 & 0.366 \\
RP & $91.41 \pm 21.95$ & $91.85 \pm 22.7$ & $90.32 \pm 20.3$ & 0.587 & 0.557 \\
BP & $74.64 \pm 19.72$ & $74.96 \pm 20.9$ & $73.86 \pm 16.6$ & 0.497 & 0.619 \\
GH & $73.90 \pm 17.32$ & $75.92 \pm 17.29$ & $68.97 \pm 16.44$ & 3.629 & $0.001^{*}$ \\
VT & $49.72 \pm 15.22$ & $40.01 \pm 12.4$ & $48.13 \pm 15.50$ & 7.382 & $0.001^{*}$ \\
SF & $85.58 \pm 17.35$ & $86.90 \pm 17.62$ & $82.37 \pm 16.32$ & 2.345 & $0.020^{*}$ \\
RE & $86.15 \pm 22.92$ & $87.67 \pm 23.40$ & $82.44 \pm 21.4$ & 2.041 & $0.042^{*}$ \\
MH & $43.68 \pm 11.88$ & $42.59 \pm 10.89$ & $46.33 \pm 13.70$ & 2.831 & $0.005^{*}$ \\
PCS & $80.95 \pm 12.03$ & $81.48 \pm 12.85$ & $79.65 \pm 9.65$ & 1.358 & 0.175 \\
MCS & $66.28 \pm 9.12$ & $67.58 \pm 7.88$ & $63.12 \pm 11.02$ & 4.461 & $0.001^{*}$ \\
\hline
\end{tabular}

* Significant differences

\section{Key:}

PF: Physical Functioning; RP: Role Limitations due to Physical Problems; BP: Bodily Pain; GH: General Health; VT: Vitality; SF: Social Functioning; RE: Role Limitation due to Emotional Problems; MH: Mental Health; PCS: Physical Component Summary; MCS: Mental Component Summary

Table III: One way ANOVA comparison of Respondents score on SF-36 by age groups

\begin{tabular}{|c|c|c|c|c|c|c|c|}
\hline \multirow[t]{2}{*}{ Subscales } & \multicolumn{5}{|c|}{ Age groups (years) } & \multirow[b]{2}{*}{ F-ratio } & \multirow[b]{2}{*}{ p-value } \\
\hline & $\begin{array}{l}20-29 \\
\bar{x} \pm S D \\
(n=160)\end{array}$ & $\begin{array}{l}30-39 \\
\bar{x} \pm S D \\
(n=146)\end{array}$ & $\begin{array}{l}40-49 \\
\bar{x} \pm S D \\
(n=74)\end{array}$ & $\begin{array}{l}50-59 \\
\bar{x} \pm S D \\
(n=3)\end{array}$ & $\begin{array}{l}60-69 \\
\bar{x} \pm S D \\
(n=2)\end{array}$ & & \\
\hline$\overline{\mathrm{PF}}$ & $82.19 \pm 21.99$ & $91.23 \pm 18.57$ & $73.58 \pm 20.20$ & $55.00 \pm 0.0$ & $95.00 \pm 0.0$ & 11.492 & $0.001 *$ \\
\hline RP & $92.50 \pm 18.78$ & $89.04 \pm 28.12$ & $93.24 \pm 13.28$ & $100.00 \pm 0.0$ & $100.00 \pm 0.0$ & 0.842 & 0.499 \\
\hline $\mathrm{BP}$ & $74.41 \pm 13.30$ & $71.68 \pm 23.78$ & $81.01 \pm 21.16$ & $57.50 \pm 0.0$ & $100.00 \pm 0.0$ & 4.299 & $0.002 *$ \\
\hline $\mathrm{GH}$ & $80.00 \pm 18.12$ & $69.78 \pm 16.85$ & $59.19 \pm 14.0$ & $45.00 \pm 0.0$ & $60.00 \pm 0.0$ & 10.455 & $0.001 *$ \\
\hline VT & $49.80 \pm 13.99$ & $46.10 \pm 14.23$ & $56.93 \pm 17.57$ & $43.75 \pm 0.0$ & $50.00 \pm 0.0$ & 6.701 & $0.001 *$ \\
\hline SF & $89.61 \pm 16.84$ & $83.82 \pm 17.50$ & $81.25 \pm 16.19$ & $87.50 \pm 0.00$ & $50.00 \pm 0.0$ & 0.642 & $0.001 *$ \\
\hline RE & $84.79 \pm 16.65$ & $86.76 \pm 24.91$ & $86.94 \pm 30.13$ & $100.00 \pm 0.0$ & $100.00 \pm 0.0$ & 0.642 & 0.633 \\
\hline MH & $43.13 \pm 13.73$ & $45.98 \pm 10.43$ & $41.80 \pm 7.93$ & $28.89 \pm 0.0$ & $11.11 \pm 0.0$ & 7.287 & $0.001 *$ \\
\hline PCS & $82.27 \pm 6.89$ & $80.43 \pm 16.76$ & $79.40 \pm 8.91$ & $65.63 \pm 0.0$ & $92.50 \pm 0.0$ & 2.579 & $0.037 *$ \\
\hline MCS & $66.83 \pm 7.67$ & $65.67 \pm 9.16$ & $66.73 \pm 11.68$ & $65.03 \pm 0.0$ & $52.78 \pm 0.0$ & 1.473 & 0.210 \\
\hline
\end{tabular}

*Significant difference

Key:

PF: Physical Functioning; RP: Role Limitations due to Physical Problems; BP: Bodily Pain; GH: General Health; VT: Vitality; SF: Social Functioning; RE: Role Limitation due to Emotional Problems; MH: Mental Health; PCS: Physical Component Summary; MCS: Mental Component Summary

Master's degree holders scored highest on BP, VT, RE subscales and PCS ( $\mathrm{p}<0.01)$ (Table IV); while those with PhD scored highest on $\mathrm{PF}$ and $\mathrm{MH}$ subscales ( $\mathrm{p}<0.01$; partial eta squared ranged from 0.02 to 0.25 ). Significant differences also exist among the different professionals on all SF 36 subscales as well as PCS and MCS ( $<<0.01$; eta squared 0.06 to 0.27 ) (Table V). Physiotherapists, the least number of health professionals surveyed $(18,4.7 \%)$ had highest scores on PF, RP, BP, SF subscales, PCS and MCS ( $<<0.01)$ while Laboratory Scientists $(32,8.3 \%)$ scored lowest on BP, 
GH and SF subscales $(\mathrm{p}<0.01)$ (Table 5). Respondents between 11- and 20-years work experience scored highest on PF and SF subscales and MCS, while those on 21-30 years score the highest on PF, GH and MH subscales ( $p<0.05$; partial eta squared ranged from 0.03 to 0.28 ) (Table VI).

SF-36 subscales scores by marital status showed that single respondents had significantly higher scores in 5 subscales as well as PCS $(83.59 \pm 7.29)$ and MCS $(67.89 \pm 6.90)(\mathrm{p}<0.05)$ than married respondents. Christians scored higher on RP (93.50 \pm 19.54$)$, and SF (86.18 \pm 23.3$)$ subscales than Muslim respondents, who had higher scores on BP $(75.96 \pm 19.19)$ subscale only $(\mathrm{p}<0.05)$. Other religions who are the minority scored highest only on GH $(75.00 \pm 0.00)$ subscale. There were significant differences between social status and BP, VT, SF, RE, MH subscales ( $\mathrm{p}<0.05)$ and MCS. Respondents who smoke scored higher on SF $(91.80 \pm 15.75)$ subscale only while those who take alcohol had higher scores on four subscales and MCS $(71.59 \pm 7.32)(\mathrm{p}<0.05)$. Significant differences exist on RP, BP, GH, RE,

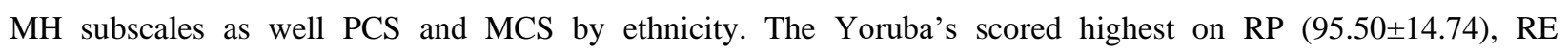
(89.39 \pm 19.52$)$, BP $(77.97 \pm 17.92)$ subscales as well as PCS (82.55 \pm 8.49$)$, the Igbos on GH $(76.93 \pm 20.94)$, while another ethnicity who are a minority (3\%) scored highest on MH (51.30 \pm 13.04$)$ and MCS (68.08 \pm 6.14$)(\mathrm{p}<0.05)$.

Table IV: One way ANOVA comparison of respondents scores on SF-36 by educational qualifications

\begin{tabular}{|c|c|c|c|c|c|c|}
\hline $\begin{array}{l}\text { SF-36 } \\
\text { Subscales }\end{array}$ & $\begin{array}{l}\text { Registered } \\
/ \text { Midwives } \\
\overline{\mathbf{x}} \pm \operatorname{SD}(\mathbf{n}=55)\end{array}$ & $\begin{array}{l}\text { Bachelor's degree } \\
\overline{\mathbf{x}} \pm \operatorname{SD}(n=275)\end{array}$ & $\begin{array}{l}\text { Master's } \\
\text { degree } \\
\bar{x} \pm \operatorname{SD}(n=52)\end{array}$ & $\begin{array}{l}\text { Ph. D } \\
\overline{\mathbf{x}} \pm \mathbf{S D}(n=3)\end{array}$ & F-ratio & p-value \\
\hline $\mathrm{PF}$ & $94.73 \pm 4.85$ & $83.33 \pm 22.05$ & $73.85 \pm 23.90$ & $95.00 \pm 0.00$ & 7.229 & $0.001 *$ \\
\hline $\mathrm{RP}$ & $94.55 \pm 10.42$ & $89.42 \pm 25.15$ & $98.08 \pm 6.73$ & $87.22 \pm 8.4$ & 2.225 & 0.066 \\
\hline BP & $73.86 \pm 18.50$ & $70.99 \pm 18.99$ & $94.09 \pm 12.78$ & $77.50 \pm 0.00$ & 18.222 & $0.001 *$ \\
\hline $\mathrm{GH}$ & $68.86 \pm 14.99$ & $75.64 \pm 18.58$ & $70.91 \pm 10.42$ & $62.50 \pm 0.00$ & 66.701 & $0.029 *$ \\
\hline VT & $35.68 \pm 13.26$ & $49.82 \pm 13.67$ & $64.18 \pm 11.22$ & $43.75 \pm 0.00$ & 31.212 & $0.001 *$ \\
\hline SF & $80.00 \pm 17.77$ & $86.72 \pm 17.87$ & $85.34 \pm 13.50$ & $87.50 \pm 0.00$ & 1.748 & 0.139 \\
\hline RE & $76.97 \pm 15.55$ & $86.98 \pm 22.93$ & $92.31 \pm 26.91$ & $66.67 \pm 0.00$ & 3.989 & $0.003 *$ \\
\hline MH & $48.04 \pm 10.78$ & $43.47 \pm 12.42$ & $38.76 \pm 5.59$ & $68.89 \pm 0.00$ & 8.053 & $0.001 *$ \\
\hline PCS & $83.00 \pm 5.89$ & $79.85 \pm 13.39$ & $84.23 \pm 8.26$ & $83.75 \pm 0.00$ & 2.173 & 0.071 \\
\hline MCS & $60.17 \pm 9.34$ & $66.75 \pm 8.84$ & $70.15 \pm 7.65$ & $66.70 \pm 0.00$ & 9.582 & $0.001 *$ \\
\hline
\end{tabular}

* Significant differences

Key:

PF: Physical Functioning; RP: Role Limitations due to Physical Problems; BP: Bodily Pain; GH: General Health; VT: Vitality; SF: Social Functioning; RE: Role Limitation due to Emotional Problems; MH: Mental Health; PCS: Physical Component Summary; MCS: Mental Component Summary 
Table V: One way ANOVA comparison of respondents scores on SF-36 by profession

\begin{tabular}{|c|c|c|c|c|c|c|c|c|}
\hline \multirow[b]{3}{*}{$\begin{array}{l}\text { SF-36 } \\
\text { Subscales }\end{array}$} & \multicolumn{8}{|c|}{ Health Professionals } \\
\hline & $\begin{array}{l}\text { Medical } \\
\text { Doctors }\end{array}$ & Nurses & Pharmacists & $\begin{array}{l}\text { Radio- } \\
\text { graphers }\end{array}$ & $\begin{array}{l}\text { Medical } \\
\text { Laboratory } \\
\text { Scientists }\end{array}$ & $\begin{array}{l}\text { Physio- } \\
\text { therapists }\end{array}$ & & \\
\hline & $\overline{\mathbf{x}} \pm \operatorname{SD}(n=168)$ & $\overline{\mathbf{x}} \pm \operatorname{SD}(n=73)$ & $\begin{array}{l}\overline{\mathbf{x}} \pm \text { SD } \\
(\mathrm{n}=53)\end{array}$ & $\begin{array}{l}\overline{\mathbf{x}} \pm \text { SD } \\
(\mathrm{n}=41)\end{array}$ & $\overline{\mathbf{x}} \pm \mathrm{SD}(\mathrm{n}=32)$ & $\begin{array}{l}\overline{\mathbf{x}} \pm \mathrm{SD} \\
(\mathrm{n}=18)\end{array}$ & F ratio & p-value \\
\hline PF & $75.42 \pm 23.20$ & $92.47 \pm 9.10$ & $87.64 \pm 17.1$ & $94.88 \pm 14.98$ & $80.16 \pm 29.50$ & $97.22 \pm 10.60$ & 14.056 & $0.001 *$ \\
\hline $\mathrm{RP}$ & $91.22 \pm 19.32$ & $95.89 \pm 9.33$ & $100.00 \pm 0.00$ & $78.05 \pm 40.39$ & $80.47 \pm 32.8$ & $100.00 \pm 0.00$ & 8.093 & $0.001 *$ \\
\hline $\mathrm{BP}$ & $79.24 \pm 17.58$ & $74.55 \pm 16.88$ & $79.20 \pm 10.51$ & $58.84 \pm 12.41$ & $51.48 \pm 26.23$ & $95.83 \pm 14.85$ & 27.872 & $0.001 *$ \\
\hline GH & $76.56 \pm 18.82$ & $68.15 \pm 14.14$ & $77.83 \pm 13.12$ & $82.01 \pm 10.10$ & $55.47 \pm 18.50$ & $75.00 \pm 0.00$ & 14.056 & $0.001 *$ \\
\hline VT & $54.13 \pm 14.71$ & $34.76 \pm 13.70$ & $46.58 \pm 14.01$ & $56.25 \pm 1.98$ & $57.42 \pm 2.94$ & $50.00 \pm 0.00$ & 27.663 & $0.001 *$ \\
\hline SF & $84.08 \pm 17.18$ & $82.02 \pm 15.86$ & $91.27 \pm 13.11$ & $87.81 \pm 21.38$ & $81.64 \pm 21.06$ & $99.31 \pm 2.95$ & 4.969 & $0.011^{*}$ \\
\hline $\mathrm{RE}$ & $93.85 \pm 22.08$ & $77.17 \pm 15.59$ & $96.23 \pm 10.66$ & $66.67 \pm 25.82$ & $66.67 \pm 23.95$ & $42.22 \pm 6.50$ & 26.603 & $0.001 *$ \\
\hline MH & $39.14 \pm 12.14$ & $46.39 \pm 10.82$ & $44.95 \pm 8.81$ & $52.25 \pm 13.34$ & $50.56 \pm 4.19$ & $39.51 \pm 5.53$ & 14.934 & $0.001 *$ \\
\hline PCS & $80.61 \pm 8.90$ & $82.77 \pm 6.35$ & $82.77 \pm 4.64$ & $78.45 \pm 15.42$ & $66.89 \pm 23.84$ & $92.01 \pm 6.36$ & 17.632 & $0.001 *$ \\
\hline MCS & $67.80 \pm 9.36$ & $60.09 \pm 8.74$ & $69.76 \pm 4.88$ & $65.74 \pm 8.43$ & $64.07 \pm 10.01$ & $72.20 \pm 0.75$ & 12.837 & $0.001 *$ \\
\hline
\end{tabular}

* Significant differences

Key:

PF: Physical Functioning; RP: Role Limitations due to Physical Problems; BP: Bodily Pain; GH: General Health;

VT: Vitality; SF: Social Functioning; RE: Role Limitation due to Emotional Problems; MH: Mental Health; PCS:

Physical Component Summary; MCS: Mental Component Summary

Table 6: One way ANOVA comparison of respondents' scores on SF-36 subscales by number of years in the profession

\begin{tabular}{|c|c|c|c|c|c|c|}
\hline \multicolumn{5}{|c|}{ SF-36 Odetunde et al., 2021} & \multicolumn{2}{|c|}{ OJMR 2(2) | 83} \\
\hline Subscales & $\overline{\mathbf{x}} \pm \operatorname{SD}(\mathrm{n}=\mathbf{3 0 0})$ & $\overline{\mathbf{x}} \pm \operatorname{SD}(n=79)$ & $\overline{\mathbf{x}} \pm \operatorname{SD}(\mathbf{n}=2)$ & $\overline{\mathbf{x}} \pm \mathbf{S D}(\mathrm{n}=4)$ & & \\
\hline $\mathrm{PF}$ & $82.08 \pm 22.98$ & $90.38 \pm 12.53$ & $85.00 \pm 7.07$ & $83.75 \pm 19.31$ & 3.194 & $0.024 *$ \\
\hline $\mathrm{RP}$ & $89.45 \pm 23.80$ & $98.58 \pm 11.32$ & $87.50 \pm 17.68$ & $100.00 \pm 0.00$ & 3.906 & $0.009 *$ \\
\hline $\mathrm{BP}$ & $73.64 \pm 21.00$ & $76.85 \pm 15.80$ & $68.75 \pm 13.40$ & $80.00 \pm 19.60$ & 0.700 & 0.553 \\
\hline GH & $75.92 \pm 18.03$ & $66.13 \pm 11.52$ & $81.25 \pm 26.52$ & $71.88 \pm 15.73$ & 7.107 & $0.001 *$ \\
\hline VT & $50.42 \pm 15.13$ & $47.78 \pm 15.51$ & $37.50 \pm 17.68$ & $42.19 \pm 11.83$ & 1.396 & 0.243 \\
\hline SF & $84.17 \pm 18.38$ & $91.77 \pm 1.38$ & $87.50 \pm 0.00$ & $68.75 \pm 21.65$ & 5.463 & $0.001 *$ \\
\hline RE & $86.00 \pm 24.47$ & $86.50 \pm 16.46$ & $83.33 \pm 23.57$ & $91.67 \pm 16.67$ & 0.097 & 0.962 \\
\hline MH & $43.02 \pm 11.72$ & $47.03 \pm 10.99$ & $51.11 \pm 15.71$ & $22.78 \pm 14.21$ & 7.115 & $0.001 *$ \\
\hline PCS & $80.37 \pm 13.02$ & $82.87 \pm 7.07$ & $84.06 \pm 2.21$ & $84.84 \pm 12.93$ & 1.092 & 0.352 \\
\hline MCS & $65.90 \pm 9.42$ & $68.27 \pm 7.45$ & $64.86 \pm 14.24$ & $56.35 \pm 5.87$ & 3.073 & $0.028 *$ \\
\hline
\end{tabular}

*Significant difference

\section{DISCUSSION}

This study constitutes one of the very few attempts to assess HRQOL of a healthy population in Nigeria and one of the first of such attempts among health professionals. This is in line with the earlier report of the dearth of studies on HRQOL among adults of the general population who are non-institutionalized and those with no specific pathology 
(Noronha et al, 2016). The response rate of this study was very high as all 385 health professionals approached completed the questionnaire. Respondents scored well above the cut-off point average quality of life on most SF-36 subscales with better physical health than mental health. This is better than the low overall HRQOL reported in a similar study by Jovic-Vranes et al. (2008) in Serbian healthcare workers and average overall QOL by Kumar et al. (2018) among health professionals in a multispecialty tertiary hospital.

Thus, our sample of health professionals which comprised the male majority in the 20-39-year age group may be said to have satisfactory health status reflecting mild to moderate effect of age on the HRQOL. This is different from the female majority reported in earlier studies (Guler and Kuzu 2009; Kumar et al., 2018) and may be attributed to involving only nurses and physicians in those studies whereas our study involved broader categories of health professionals. The gender difference in the HRQOL of the respondents in favour of male health professionals is consistent with previous studies (Tountas et al, 2007; Saridi et al, 2019). This seems to reflect the disadvantaged position of females in a male societal structure and working environment in several nations (Guler and Kuzu, 2009). Additional roles of females in the nuclear and extended family settings, religious centres and the society at large in this environment may constitute some level of burden and consequently lower their HRQOL. Incidentally, Nigerians are believed to be very religious, with the females more spiritual than the males.

However, this does not translate to appreciable HRQOL both in the physical and mental health of respondents in this study. As expected in a setting of very religious people, good social habits were reported among the respondents as majority neither smokes nor takes alcohol, similar to the report of Kumar et al., (2018). This may be also be attributed to the acquired knowledge of health professionals on adverse effects of risky social habits which they, as role models in the general community should exemplify in their health practices and patient counselling (Pistikou $e t$ $a l$, 2014). Even at that, few respondents who smoke (8.3\%) and take alcohol (4.9\%) still demonstrated better HRQOL in more subscales and mental health. This proportion is however too small to justify better HRQOL among smokers and alcohol drinkers. The reported age range of the majority of the respondents in this study is an indication that larger proportions of health professionals are below 40 years old in line with earlier studies (Guler and Kuzu 2009; Barbosa et al, 2018). The youngest age group had better HRQOL compared with other age groups, in line with an earlier finding (Kheiraoui et al, 2012). Surprisingly, the oldest age group (60-69 years) reported comparable HRQOL in addition to having the highest physical component score (PCS), which indicates a better state of physical health than their younger counterparts. This should however be treated with caution due to the very small sample size of the oldest age group. Over half of the respondents were married but HRQOL was generally better in the single respondents in most subscales. An indication of better physical and mental health status among the single respondents may be associated with a higher level of family commitment among married respondents which may constitute an additional burden and lower HRQOL. This may explain why single respondents in this study had better HRQOL. The influence of ethnicity on HRQOL cannot be overemphasized in a multi-ethnic nation like Nigeria. This was demonstrated by better HRQOL in more subscales and physical health of Yoruba respondents, the major ethnic group in southwestern Nigeria where this study was conducted.

Bachelor's degree is the entry point for job placement in the scheme of service for most health professionals in Nigeria and this explains why the majority of our respondents are in this category. The educational qualification of 
our respondents is shown to have a small to large effect on their HRQOL as indicated in the reported effect size. However, quite a number had postgraduate qualifications (14\%), a proportion higher than $6.5 \%$ reported by Kumar et al., (2018). This translated to better HRQOL scores and better physical health of the respondents in our study, similar to the findings of Saridi et al, (2019). Health professionals at different grade levels had similar HRQOL although those in the highest grade level category had the best mental health. This may indicate that higher or lower grade level category may not necessarily translate to a better quality of life. This finding may also be attributed to higher workloads usually experienced by junior professionals or unsatisfactory work situations and administrative bottlenecks frequently faced by workers in this environment. The professional status of the participants in this study reveals notable health inequalities among the health professionals, with an indication of a small to large implication on their HRQOL. This shows that HPs in this study are far from constituting a homogenous group of professionals with similar health status and health-related quality of life, similar to the conclusion of Kheiraou et al. (2012).

Physiotherapists and Laboratory Scientists at the extreme opposite ends of HRQOL may not be expected to produce any logical conclusion as their numbers are far less than other professionals. On the other hand, however, the high HRQOL may reflect the effect of knowledge and role of physiotherapists in promoting health and wellness through physical activity, exercises, healthy lifestyle and disease prevention activities involved in their practice. HRQOL of the radiographers and the medical laboratory scientists are generally low which may reflect unsatisfactory working conditions, workload and quality of life. The medical doctors and pharmacists had satisfactory HRQOL with pharmacists reflecting better mental health. Long or short years of experience of health professionals in this study did not necessarily translate to a better quality of life which may also be an indication of unsatisfactory work conditions and/or remuneration.

\section{CONCLUSION}

Our study revealed that considerable inequalities in HRQOL exist among health professionals. These inequalities are gender-biased and favour professionals with a post-graduate degree. Findings from this study are comparable to reports from other climes and advocate support for female health professionals, those on low cadre and encourage higher degree training. This study provided an impetus for further studies in multiple health care settings in different areas of the country and other LMIC to plan future directions of health-related developments.

\section{LIMITATION OF THE STUDY}

This study has some limitations. The self-report measurement used to assess the HRQOL of health professionals in this study might have subjected it to response bias, so the researcher had to assume that the respondents were truthful. This study was limited by the varying numbers of the different health professionals that made up the sample. The results may not indicate a true measure of HRQOL for the professionals such as physiotherapists with a comparatively small number of respondents.

\section{CONFLICTS OF INTERESTS}

The authors declare that they have no competing interests. 


\section{ACKNOWLEDGEMENTS}

We are grateful to the health professionals who participated in this study.

\section{ABBREVIATIONS}

HRQOL- Health Related Quality of Life LMIC-Lower Middle-Income Economy MCS- Mental Component Summary

HP- Health Professionals SF 36- Short form 36-item health survey PCS-Physical Component Summary

\section{REFERENCES}

Acaray PR, Pinar R. (2004). Assessment of quality of life in chronic hemodialysis patients. Cumhuriyet University Journal of Nursery Academy. 8:1-11

Barbosa ML, Menezes TN, Santos SR, Olinda RA, Costa GM. (2018). The quality of life of health professionals working in the prison system. Ciência \& Saúde Coletiva. 23 (4): 1293-302

Centre for Disease Control (CDC): United States of America National Center for Chronic Disease Prevention and Health Promotion Division of Population Health. https://www.cdc.gov/HRQOL/methods.htm Accessed on 28/01/2021, Accessed on 20 March 2021

Cohen, J. (1988). Statistical Power Analysis for the Behavioral Sciences. New York, NY: Routledge Academic. Retrieved on 5 July, 2021 from:https://www.frontiersin.org/articles/10.3389/fpsyg.2013.00863/full

Deaton, A.S. and Tortora, R., 2015. People in sub-Saharan Africa rate their health and health care among the lowest in the world. Health Affairs, 34(3):519-527.

Gandek B, Sinclair SJ, Kosinski M, Ware Jr JE. (2004). Psychometric evaluation of the SF-36® health survey in Medicare managed care. Health Care Financing Review. 25(4):5-25.

Guler N, Kuzu F. (2009). The health-related quality of life of the health professionals working in the primary healthcare centers and its correlation with selected socio-demographic factors in Sivas, a central Anatolian city. Scientific Research and Essays. 31; 4(12):1547-52.

Gurses AP, Carayon P, Wall M. (2009). Impact of performance obstacles on intensive care nurses' workload, perceived quality and safety of care, and quality of working life. Health Services Research. 44(2p1):42243.

Jovic-Vranes A, Vesna B, Boris V, Natasa M. (2008). Job satisfaction in Serbian health care workers who work with disabled patients. Open Medicine. 3(2):221-4.

Kheiraoui F, Gualano MR, Mannocci A, Boccia A, La Torre G. (2012). Quality of life among healthcare workers: a multicentre cross-sectional study in Italy. Public Health. 126 (7):624-9. https://doi.org/10.1016/j.puhe.2012.03.006 Accessed on 24 July 2020

Kumar A, Bhat PS, Ryali S. (2018). Study of quality of life among health workers and psychosocial factors influencing it. Industrial Psychiatry Journal. 27(1):96. 
Landman GW, Van Hateren KJ, Kleefstra N, Groenier KH, Gans RO, Bilo HJ. (2010). Health-related quality of life and mortality in a general and elderly population of patients with type 2 diabetes (ZODIAC-18). Diabetes Care 33(11):2378-82

Lee PH, Chang WY, Liou TH, Chang PC (2006). Stage of exercise and health-related quality of life among overweight and obese adults. Journal of Advanced Nursing 53(3):295-303 https://doi.org/10.1111/j.13652648.2006.03726.x

Mc Vicar A. (2003). Workplace stress in nursing: a literature review. Journal of Advanced Nursing 44(6):633-42.

Mujchin IG. (2015). Quality of Life of the Health Care Workers in the Pre-Retirement Period from the Private Sector of the Primary Health Care from the Skopje Region. Open access Macedonian Journal of Medical Sciences. 3(3):514-520.

Müller H, Franke A, Resch KL. (2007). Application of the German SF-36 in hospitals: Overestimations in the psycho-social scales. International Journal of Public Health 52(1):60. https://doi.org/10.1007/s00038-0065091-9 Accessed on 25 April 2020

Noronha DD, Martins AM, Dias DD, Silveira MF, Paula AM, Haikal DS. (2016). Factors in adult health-related quality of life: a population-based study. Ciência \& Saúde Coletiva 21:463-74. https://doi.org/10.1590/141381232015212.01102015, Accessed on 18 April 2020

Nylenna M, Gulbrandsen P, Førde R, Aasland OG. (2005). Unhappy doctors? A longitudinal study of life and job satisfaction among Norwegian doctors 1994-2002. BMC Health Services Research. 5 (1):44. https://doi.org/10.1186/1472-6963-5-44 Accessed on 18 April 2020

Oni OD, Aina OF, Ojini FI, Olisah VO. (2016). Quality of life and associated factors among poststroke clinic attendees at a University Teaching Hospital in Nigeria. Nigerian Medical Journal: Journal of the Nigeria Medical Association. 57(5):290.

Oyama Y., Yonekura Y, Fukahori H. (2015) Nurse health related quality of life: associations with patient and ward characteristics in Japanese general acute care wards. Journal of Nursing Management. https://doi.org/10.1590/1413-81232018234.09292016 Accessed on 27 April 2020

Palhares VD, Corrente JE, Matsubara BB. (2014). Association between sleep quality and quality of life in nursing professionals working rotating shifts. Revista de Saude Publica. 48:594-601.

Phan HT, Blizzard CL, Reeves MJ, Thrift AG, Cadilhac DA, Sturm J, Heeley E, Otahal P, Rothwell P, Anderson CS, Parmar P (2019). Sex differences in long-term quality of life among survivors after stroke in the INSTRUCT. Stroke. 50(9):2299-306.

Pistikou AM, Zyga S, Sachlas A, Efthymia Katsa M, Daratsianou M, Andrea Paola RG. (2014) Determinative factors of being an effective health-care role model. International Journal of Occupational Health and Public Health Nursing 1(3):3-14.

Saridi M, Filippopoulou T, Tzitzikos G, Sarafis P, Souliotis K, Karakatsani D. (2019). Correlating physical activity and quality of life of healthcare workers. BMC Research Notes. 12(1):208-213. https://doi.org/10.1186/s13104-019-4240-1 Accessed on 30 April 2020 
ten Klooster PM, Vonkeman HE, Taal E, Siemons L, Hendriks L, de Jong AJ, Dutmer EA, van Riel PL, van de Laar MA (2013). Performance of the Dutch SF-36 version 2 as a measure of health-related quality of life in patients with rheumatoid arthritis. Health and Quality of Life Outcomes. 11(1):1-9.

Tinubu BM, Mbada CE, Oyeyemi AL, Fabunmi AA. (2010). Work-related musculoskeletal disorders among nurses in Ibadan, South-west Nigeria: a cross-sectional survey. BMC Musculoskeletal Disorders. 1;11(1):1-8.

Tountas Y, Manios Y, Dimitrakaki C, Tzavara C. (2007). Relationship between basic protective health behaviours and health related quality of life in Greek urban hospital employees. International Journal of Public Health. 1; 52(6):341-7.

WHO Work Plan 2009-2012. WHO Global Network of Collaborating Centres in Occupational Health. Available at: www.who.int/occupational_health Accessed on: 17.07.2015

WHO work plan on climate change and health 2014-2019 https://www.who.int/globalchange/health_policy/en/]. Accessed on 11 April, 2020 